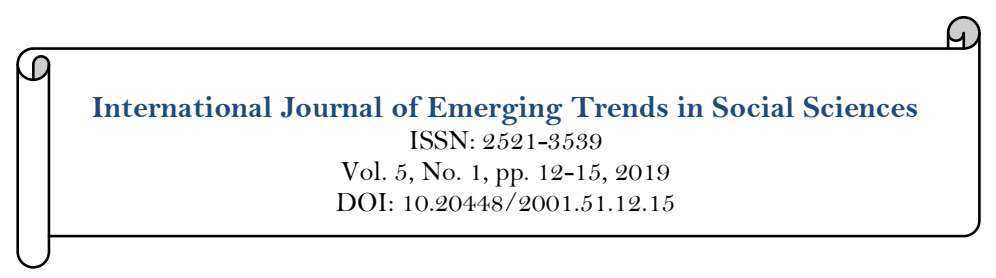

$\mathcal{V}$ updates

\title{
Design of GPS Information Processing System Based on Single Chip Microcomputer
}

\author{
Yu Zhao's \\ Yanqing $\mathbf{Z h a n}^{2}$ \\ Hongbing Zhou ${ }^{3}$ \\ 'Jiangxi Normal University, Management Science and Engineering Research Center, Ziyang Road No.99, Nanchang, Jiangxi Province, China. \\ Email: srinunaikphd@gmail.com \\ Jiangxi Normal University, College of History, Culture and Tourism, Jiangxi Normal University, Ziyang Road No.99, Nanchang, Jiangxi \\ Province, China. \\ "Jiangxi Normal University, College of City Construction, Jiangxi Normal University, Ziyang Road No.99, Nanchang, Jiangxi Province, \\ China.
}

\section{Abstract}

With the rapid development of science and technology in the 21st century, the Global Positioning System (GPS) has become one of the representatives of the development achievements of this era. The system not only has high precision and good performance, but also has a wide range of application. It has been widely used in land, ocean, space and other fields, with a very high market share. In response to a surge in demand for satellite-positioning and navigation applications, In this paper, the GPS information processing system is constructed with hardware modules such as GPS receiving module and LCD module as the main structure, single chip microcomputer as the core controller, supplemented by necessary peripheral circuits. The success of GPS information processing system based on single chip microcomputer solves the problem of high price of GPS devices in the market. In general, the design structure is not only modular and easy to carry, the most important is the human nature, cost-effective, and has great practical value.

Keywords:
GPS
Single chip microcomputer
Information processing system.
Licensed:
This work is licensed under a
Creative Commons Attribution 4.0
License.
Publisher:
Scientific Publishing Institute

Keywords:

Single chip microcomputer

This work is licensed under a

Creative Commons Attribution 4.0

Publisher:

Scientific Publishing Institute

Funding: This study received no specific financial support.

Competing Interests: The authors declare that they have no competing interests.

\section{Introduction}

With the development of cloud computing, big data, Internet of things and $5 \mathrm{G}$ technology, the application demand of satellite positioning and navigation is surging, and its scale and service type are also more extensive. The realization of all-weather, all-terrain and real-time positioning has become particularly important. The GPS information processing system based on single chip microcomputer has this function.

\subsection{Background and Significance of the Design}

Global Positioning System (GPS), the English translation of the Global Positioning System, the predecessor of the U.S. maritime force for the ocean navigation System, is the product of the follow-up development of Transit. It has the characteristics of full time, global and real-time. Because of its high accuracy, strong reliability and wide coverage, it is now the most popular and familiar satellite navigation and positioning system used in positioning and navigation services (Mingliang \& Qianhui, 2009).

So did in China, scientists, and other countries as positive development in the use of GPS signal resource at the same time, also is committed to research and development its own satellite positioning system, such as Europe's Galileo satellite positioning system, built in the Russian glonass satellite navigation system, China is also committed to the development of beidou satellite navigation and positioning system to herself, and progress rapidly, by early 2019 , has basic global coverage in advance. 
Nowadays, GPS is widely used. At the present stage, the main development trend is that the application field has been flowing to the land market from the traditional range of air and sea, among which the main applications can be summarized as land, sea, space and other aspects.

\subsection{Existing Problems}

GPS is the most important signal modulation and output, at the same time, can accurately show the latitude and longitude coordinates, altitude, time and moving speed of the observation point, also directly affects the practical value of GPS satellite positioning navigation system. GPS navigation does have a powerful function, because it is a sophisticated industry, so the disadvantages are expensive, circuit multifusing. When GPS is used for mobile navigation and positioning, its two conditions limit their availability: line-of-sight connection, that is, the connection between GPS terminal and satellite should be realized; Information about the orbit, parameters and ephemeris of the satellite (Tianwen, 2010).

\subsection{The Future Development Trend of the Industry}

In recent years, the application of satellite navigation in mobile terminals, transportation industry, Internet of things and other aspects has grown particularly rapidly. Relying on cloud computing, big data, Internet + and $5 \mathrm{G}$ technology, the application scope of satellite navigation is huge in terms of scale and service type. In the coming decades, the market will keep growing. Thus, the potential application prospect of GPS technology is bright (Xiaofei, Chengfa, Shuguo, \& Weirong, 2013).

\subsection{The Main Content of the System Design}

This design combines single chip microcomputer and satellite navigation and positioning knowledge, and designs a system composed of single chip microcomputer chip, GPS signal receiving module and 12864LCD module. The satellite signal is received and extracted through the global positioning system signal receiving module, which forms the core part with the single chip microcomputer, conducts signal modulation, analysis, and USES the LCD liquid crystal display module to accurately display the required real-time data. Finally, on the basis of the completion of schematic diagram design, professional software Proteus is used for simulation test of schematic diagram.

\section{System Design Scheme}

2.1. GPS Positioning System

GPS technology is a complex high-tech product, which is mainly composed of the following three parts according to its different functions:

(1) Ground monitoring system: including the main control base station, injection station and antenna, measurement station;

(2) Navigation satellites: twenty-four satellites are distributed in six different orbit levels in space, which are the signal sources of the global positioning system;

(3) User terminal: It is composed of satellite antenna, GPS receiving module and control display.

Working principle is: first, ground monitoring control system for the capture the navigation satellite signal to after continuous tracking satellite, these signals will be sent to the corresponding data processing equipment, then calculate the signal from the satellite to user terminal part of the time interval, pseudorange measurement, and use the formula to calculate and positioning algorithm. The whole process needs to capture no less than 4 signals broadcast by static satellites. Secondly, the detection of propagation time requires the synchronization clock between the receiver and the satellite to be accurate. In addition, measurement errors may be caused by changes in the transmission time of wireless signals in the ionosphere, noise, and quantization errors at the receiving end. Because of the uncertainty caused by these errors, kalman filter estimation method and least square method can be used to estimate the geographic coordinates of GPS terminals.

\subsection{GPS Signal Receiving Scheme}

After the satellite signal is captured and converted, the data is transmitted to the display module and displayed on the LCD. In addition, according to the single-chip microcomputer and the function of the GPS module, combined with the purpose of this design, circuit design reasonably, to ensure that after capture the satellite signal receiver, extraction time and date correctly and accurately calculate the observation point of latitude and longitude coordinates, elevation, and time and speed, and the information output to the LCD display module, finally complete the location information of real-time display.

\subsection{GPS Receiving Module}

As the key of the whole design, the function of GPS receiving module is to capture satellite signals. Its usual architectures include receiving antenna, frequency converter, preamplifier and parallel signal channel, central processing unit and I/O port, internal memory, etc. 
Generally, location estimation is based on the distance, direction and Angle between known and unknown points. In order to estimate the position of the GPS receiver, the GPS module must first capture the signal sent by the satellite, and then estimate the distance between the satellite in orbit and the receiving module itself by measuring the propagation time. With this distance and the satellite's position information, the GPS receiver's position can be estimated, including longitude, latitude and altitude, and the movement speed can be calculated based on time.

\subsection{Overall Scheme Design}

According to the design and planning of the system, the single-chip microcomputer is used as the core processor, the GPS signal receiving module is connected, and the LCD module is used to display the positioning data. Under comprehensive consideration, McS-51 series MCU is widely used as the controller. The NEO-6M GPS signal receiving module of u-blox company is used as the signal receiving part of GPS. Serial communication is conducted between the McS-51 single chip microcomputer and the NEO-6M GPS signal receiving module to complete the output of data signals. The specially designed program is used to filter, calculate and output the satellite signals captured by the receiving module to obtain relevant positioning information. The parallel interface of McS-51 is used to connect with LCD. The complete hardware architecture of global positioning information processing system based on single chip microcomputer is mainly composed of receiving module, control module circuit, display module circuit and power circuit. In addition, it includes some other peripheral circuits.

\section{Hardware Circuit Architecture}

\subsection{Hardware Body Architecture}

The hardware architecture of global positioning information processing system based on single chip microcomputer is mainly composed of receiving module, control module circuit, display module circuit and power circuit. In addition, it includes some other peripheral circuits. Driven by the power supply, the modules work together to locate and display. The main structure of the hardware is shown in Figure 3-1.

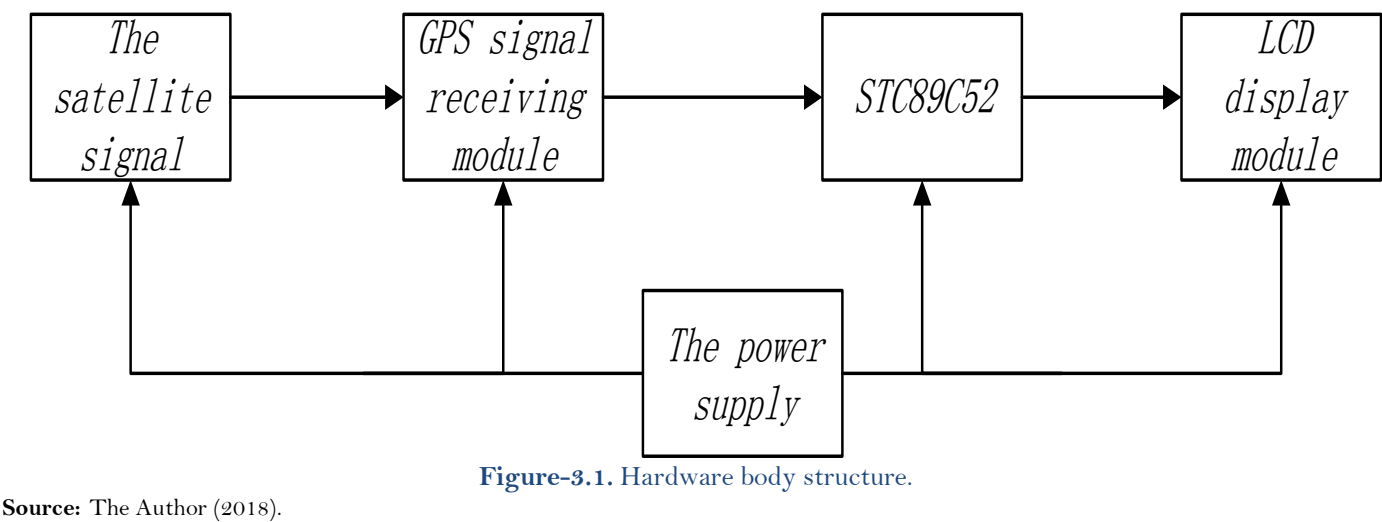

\subsection{STC89C52 Single-Chip Microcomputer}

SCM was born in the 1970s. The development speed is about every three or four years, doubling the performance and doubling the integration. Its features are full function, small appearance and high cost performance. The single chip microcomputer used in this system is STC89C52.

STC MCU is a 51 core MCU produced by shenzhen hongjing technology co., LTD., which occupies a large share In the MCU market In China. The series of MCU has low power consumption, strong interference ability and reduced electromagnetic interference performance, and ISP (In System Programming) and IAP (In Application Programming) functions. The highest working frequency of $89 \mathrm{C}$ series is $80 \mathrm{MHz}$, the RAM data memory is $512 \mathrm{~B}$ to $1280 \mathrm{~B}$, and the Flash memory is $4 \mathrm{~KB}$ to $64 \mathrm{~KB}$. The parameters of STC89C52 can achieve the direct compatibility of McS-51 operation command and transmission pin in the same industry.

\subsection{U-Blox GPS Signal Receiving Module}

The neo-6m GPS module used in the design is produced by the Swiss company u-blox, and it is the 6th generation GPS receiver. It is equipped with the core 50-channel technology of u-blox 6 , with high performance. Its effective correlative is not less than 1 million. Because use Super Sense ${ }^{\circledR}$ kick start technology, even when the signal is weak, by accelerating, still can capture a GPS satellite signal. U-blox GPS receiver has strong anti-interference ability, which benefits from the innovation of its RF architecture and the advanced noise suppression technology. At the same time, the u-blox GPS receiver also has precision timing, inertial navigation, a-gps and other technologies. 


\subsection{LCD Module 12864}

Liquid Crystal Display (Liquid Crystal Display) is a new type of Display, which is made by Liquid Crystal's twisted alignment effect. The display module used in this system is YB12864ZB, referred to as 12864 for short. 128 64 resolution, 128168 byte ASCII character sets and 8,192 1616 byte Chinese characters are built in. This module has flexible interface, convenient and concise operation instructions, friendly humancomputer interaction interface can display all Chinese, but also can display images. Low voltage and low energy consumption are the significant advantages of this module.

\section{Program Architecture}

\subsection{Programming Ideas}

How to realize the data transmission between the GPS receiving module and the single-chip microcomputer is the core problem of this design, and how to analyze and screen the information after the single-chip microcomputer receives the transmitted data information, and then send it to the 12864 LCD display module. The source program is written in $\mathrm{C}$ language.

Because this system has the modular characteristic, so in the program design also USES the block idea. This advantage is easy to write programs, convenient debugging and clear function, so the whole system program design is divided into three parts: LCD module, GPS signal receiving module, SCM module (Yuhua, $2011)$.

\subsection{Programming Flow}

The implementation process of the program is as follows. First, the STC89C52 MCU and the 12864 LCD module are initialized. The GPS receiving module will transmit the captured satellite information to the MCU. After receiving the transmitted data information, the single-chip microcomputer analyzes and screens the data such as universal time, longitude and latitude, adjusts the format at the same time, and finally sends the data to the 12864 LCD module.

\section{Software Debugging and Simulation Testing}

The purpose of software debugging is to use program debugging software and schematic diagram simulation software to debug the programmed program, simulate the drawn schematic diagram, find program errors and modify them, and at the same time, find out the hardware faults. In general, software debugging can help us better complete the hardware production and debugging. Keil uVision( $(\mathrm{V} 4)$ and Proteus software platform were used for software debugging and simulation testing.

\section{Conclusion}

The success of GPS information processing system based on single chip microcomputer solves the problem of high price of GPS devices in the market. In the process of program design and hardware debugging, modular approach, the program is more lengthy, the program has a lot of space to simplify. In view of the above problems, I will improve the system in the future study and work, and combine the change of market demand and the upgrade of GPS information processing system to continuously improve the system and give full play to its practical value.

\section{References}

Mingliang, D., \& Qianhui, T. (2009). Application design and simulation of 51 single-chip microcomputer: Based on Keil C and Proteus. Beijing: Beijing University of Aeronautics and Astronautics Press.

Tianwen, L. (2010). Principles and applications of GPS (2nd ed.). Beijing: Science Press.

Xiaofei, S., Chengfa, G., Shuguo, P., \& Weirong, C. (2013). Precision analysis of real-time service data of global positioning system. Journal of Navigation and Positioning, 1(4), 84-88.

Yuhua, L. (2011). Global positioning system technology development and domestic and foreign trends. Heilongiiang Science and Technology Information, 23(21), 68. 